\title{
On the ambiguity of visual stimulation: A reply to Eriksson
}

\author{
RICHARD R. RCSINSKI* \\ University of Pittsburgh, Pittsburgh, Pennsylvania 15260
}

\begin{abstract}
In a recent note, Eriksson criticized Gibson's theory by arguing that visual perception of distance is not solely a function of optical stimulation. It is shown here that the optic array does specify distance, and that the limitations of visual information are precisely defined in the theory. Visual information alone is insufficient when judgments in arbitrary metric units are required. The limitation of the theory is not in the specification of potential and effective information, but in the description of the observer's response to that information.
\end{abstract}

In a recent article, Eriksson (1973) raised a number of important questions concerning the nature of optical stimulation in visual perception and the adequacy of an information-based theory of perception such as developed by Gibson (1966). Briefly, Eriksson argued that "visual stimulation is characterized by a basic ambiguity [p. 381]" and that therefore an explanation of size and distance judgments based on optical information is untenable. Although Eriksson's examples of visual ambiguities are correct, it does not follow that an optically based theory of perception is necessarily faulty. It is well known that the perception of size and distance is determined by many parameters of the situation. Even though in many of his writings, Gibson has stressed the role of optical stimulation, other aspects of stimulation have not been ignored.

In fact, each of the examples Eriksson uses were discussed and amplified by Gibson and his colleagues in the course of the development of an information-based theory of perception. The ambiguities involving multiparameter influences in the perception of space form an important aspect of the mathematical theory of ecological optics. The interrelationship of these parameters in determining visual information for spatial layout has been precisely specified, and the information available from texture gradients, motion parallax, and motion perspective has been shown to affect judgments of spatial layout.

Questions involving the adequacy of optical stimulation as a basis for visual perception reduce, in part, to questions of the adequacy of these mathematical analysis of stimulus information. The purpose of the present paper is to continue the discussion of optical information and to further

\footnotetext{
*The author wishes to thank James Gibson, Donald McBurney, Bruce Goldstein, and Roberta Golinkoff for their comments on an earlier draft of the manuscript.
}

explore the strengths and limitations of an information-based theory. It is worthwhile to consider two closely related questions: first, the logical question of whether potential information exists; and second, the psychological question of whether such information is effective in perception.

\section{POTENTIAL INFORMATION}

In general, Eriksson's criticisms reduce to a single statement regarding mathematical analysis: Each form of stimulus information can be expressed as a single equation containing two or more unknown parameters. This fact has not escaped other theorists. Furthermore, a resolution can be deduced within the framework of Gibson's theory.

Eriksson states that texture gradients do not provide information for distance since any gradient could result from an infinite number of textural surfaces lying at different distances from the eye with correspondingly different texture element sizes. Except in books and articles intended for the general reader (e.g., Gibson, 1950, 1959), the theory underlying texture gradients has not been discussed by Gibson. The derivation and mathematical expressions of texture gradient notions were, however, presented by Purdy (1960) in a thesis conducted in Gibson's laboratory. In an analysis of gradient information forming the basis of most work on texture gradients and distance, Purdy precisely defined the relationship between gradients and distance.

If an element of texture subtends a solid angle, w, at the eye, the gradients of size $\left(G_{S}\right)$ is defined as the relative rate of change in $w$ as the line of regard is swept from the nadir to the horizon. If changes in angular size can be detected, $G_{S}$ can be extracted from the optic array. In a similar way, gradients of density $\left(G_{d}\right)$, compression $\left(G_{c}\right)$, and perspective $\left(G_{L}\right)$ can be defined. 
Once this gradient information can be picked up, the relationship between distance and the various gradients is given by:

$$
\mathrm{D}=\mathrm{H} \frac{\mathrm{G}_{\mathrm{s}}}{3}=-\mathrm{H} \frac{\mathrm{G}_{\mathrm{d}}}{3}=\mathrm{H} \mathrm{G}_{\mathrm{c}}=\mathrm{HG}_{\mathrm{L}} \text {, }
$$

where $D$ is distance from an observer, $H$ is the height of the eye above the ground, and $G_{S}, G_{d}, G_{c}$, and $G_{L}$ are the four texture gradients.

On the basis of gradient information alone, relative judgments of distance are possible. A comparison of several distances or a fractionation of distance can be made. Note further that the relationship between distance and gradient information is linear. This means that distances over a textured surface are specified to vithin a scale factor. If $\mathrm{H}$ is given, of course, absolute distance in metric units is specified.

Far from being an unresolved ambiguity, the effect of the scale factor has long been known. Gibson and Bergman (1954), for example, discuss the improvement in distance judgments as involving the learning of a scale factor. By definition, making metric judgments involves providing an arbitrary label ( $1 \mathrm{ft}, 1 \mathrm{~m}$, etc.) for a perceived extent. Clearly, a metric scale must be learned in order to make such judgments. If the requirement of an arbitrary metric unit is dropped, an observer could judge absolute distances (e.g., if this distance is 1 , then this 2 , etc.) solely on the basis of gradient information. These judgments may not correspond to conventional units, but they would be accurate, given the observer's scale.

Sedgwick (1973) has shown that height is potentially specified by the visible horizon. If an object on the ground extends two-thirds of its height over the horizon and one-third under, it can be shown that the object's height is three times the height of the observer's eye above the ground. Horizon information provides a potential scale for judgment and potential information about changes in the height of the station point.

A further informational specificity provided by texture gradients involves the slant of a surface. For any flat surface, a specific gradient (rate of change of element size) is uniquely associated with a specific slant angle (Flock, 1964). Although Flock's analysis is not constrained by the size, shape, or regularity of texture elements, a simple example of this specificity can be seen in Braunstein's (1968) extrapolation of Flock's analysis to regular, square texture elements. In this case, Slant $\Theta$, relative to the line of regard is given by the ratio of angular width and height as

$$
\operatorname{Cos} \theta=\frac{\text { angular height }}{\text { angular width }}
$$

Flock's analysis is not restricted to these conditions, but this simple example provides a description of the relationship between texture gradients and physical slant.

In his article, Eriksson points out that perceived distance is not specified by motion parallel to the ground, since a misestimation of velocity would cause a misperception of distance. It was Gibson, Olum, and Rosenblatt (1955) who first pointed this out and incorporated this fact into an information-based theory. If we define $\mathrm{D}$ as distance from a point $(\mathrm{P})$ to an observer, $\mathrm{V}$ as linear velocity or ground speed, $\delta$ as the angle between the line of locomotion and a line drawn from the observer to the point (OP), and $t$ as time, then distance is specified exactly as follows:

$$
\mathrm{D}=\frac{\mathrm{V}(\sin \delta)}{(\mathrm{d} \delta / \mathrm{dt})} .
$$

It is obvious from this equation that distance is a function of velocity.

Velocity is specified by a joint function of the optical flow field of motion perspective and the observer's altitude. To quote Gibson, Olum, and Rosenblatt (1955, p. 382), "Ground-speed and altitude are not ... independently determined by the optical information. A more rapid flow pattern may indicate either an increase in speed or a decrease in altitude." Consequently, perception of distance based on motion perspective is affected by the same factor $(\mathrm{H})$ as affects judgments based on texture gradients. Similarly, in the case of motion perspective, the flow gradient suffices for accurate judgment within the limits of parameter estimation.

Under conditions of locomotion toward a surface or object (impending collision or "looming"), perceived distance cannot be explained on the basis of the expansion pattern. Gibson and his colleagues were the first to point this out, and no one has suggested that loom information specifies distance. The article by Gibson et al (1955), cited above, is again relevant. The flow pattern is a joint function of both object velocity and distance to the surface. Consequently, without the observer's knowing velocity, the flow pattern could not specify distance. As Eq. 3 further reveals, distance is not specified for points on the line of locomotion, since $\sin \theta=0$ and $D$ is indeterminate.

The major specificity involved in looming involves time to collision, not distance to collision. In an analysis of helicopter landing, Gibson et al (1955) point out that length of time before touching down is given by the optical information in an unequivocal manner.

Schiff (1965), in analyzing loom information, comes to the same conclusion. Time to collision is 
exactly specified by the expansion pattern; if $a_{1}$ is the angular size of an object at time $t_{1}$, and $a_{2}$ is its size at time $t_{2}$, the time to collision (TC) is given by

$$
\mathrm{TC}=\frac{\mathrm{a}_{1}}{\left(\mathrm{a}_{2}-\mathrm{a}_{1} / \mathrm{t}_{2}-\mathrm{t}_{1}\right)} .
$$

Equation 4 holds only for motions of constant velocity. A general analysis for arbitrary accelerations is not available.

Eriksson also points out that spatial layout is not specified for an observer who rises from a seated to a standing position, since errors in estimation of velocity will result in errors of perception. This is a restatement of the second criticism discussed above, since motion perspective embodies a general rule which holds for motion in any direction. In this case, distance is given by Eq. 2 above. When motion is not parallel to the ground, the situation becomes slightly more complicated with the $\mathrm{d} \delta / \mathrm{dt}$ term of Eq. 3 a function of velocity, height, the angle between OP and a line from the observer to the nadir, the angle of the line of locomotion, and the line from $P$ to the nadir. The flow field specifies everything but $\mathrm{V}$ and $\mathrm{H}$, as in the preceding example.

\section{EFFECTIVE INFORMATION}

The preceding analyses show that texture gradients, velocity gradients, observer velocity, and observer height provide potential information for the perception of depth, distance, slant, and impending collision. If $\mathrm{V}$ and $\mathrm{H}$ are constant, relative judgments of distance are possible. If $\mathrm{V}$ and $\mathrm{H}$ are taken as scale factors, absolute judgments within these scales are possible. An important aspect of the evaluation of an information-based theory of perception concerns whether these parameters can be registered. There is ample evidence that indicates that they can be.

As pointed out before, the overall form of the texture gradient specifies slant. Even in the absence of other information for slant, subjects' judgments of slant based on texture gradients are in close correspondence with physical slant (Purdy, 1960; Flock, 1965). Flock has shown that when the gradient is supraliminal (above the limits of visual acuity), correspondence between physical and judged slant is quite high (bs $=0.85$ to 1.12 ). Further, a moderately high correspondence $(b=0.68)$ has been found even with first grade children (Levine, Rosinski, \& McDowell, 1973). Data collected by Purdy (1960) provides a conclusive demonstration that texture gradients provide effective information for slant. Magnification (m) alters the gradient such that the magnified slant, $\theta_{\mathrm{m}}$, is related to the actual slant, $\theta$, by

$$
\cot \theta_{m}=\frac{1}{m} \operatorname{Cot} \theta .
$$

Purdy found that with constant error eliminated, slant judgments under magnification were virtually identical to those predicted by the gradient analysis. Gibson (1950) has provided a number of demonstrations which show that if element size is altered, but the gradient is unchanged, a single planar slanted surface is seen. If the gradient is altered, a change in slant is seen.

Analysis of gradient information (e.g., Purdy, 1960) has been based on the simplifying assumption that equal size texture elements cover the surface. This analysis is applicable, however, to nonregular size elements as long as the element size has finite variance. Under such conditions, probable error is easily calculated. Flock and Moscattelli (1964) have verified a predicted increase in error with increasing element variability. The above studies show that the form of the texture gradient can be registered by an observer.

The perception of distance, in addition to requiring the observer to register a gradient, requires the ability to distinguish among points on the gradient (among relative rates of change). Relative judgments of distance, then, require comparisons of portions of the gradient. Experimental evidence indicates that even young children can judge distances along a gradient. Wohlwill (1965), using a bisection procedure, found that subjects were able to compare intervals along a texture gradient in making distance judgments. This indicates that rates of change along a gradient, in addition to the form of the gradient, can be registered.

Absolute judgments of distance require not only the ability to register rates of change in texture elements, but also the ability to use height $(\mathrm{H})$ in making a judgment. Gibson and Bergman (1954) have shown that subjects can make absolute distance judgments which improve as the scale is learned. The relationship between height and distance, however, poses an interesting problem for perceptual analysis. According to Purdy (Eq. 1), height of the station point affects perceived distance. Yet, if an observer is to maintain veridical perception over a variety of postures, one must compensate for the effect of differing heights. It appears that height does affect distance perception, but that some compensation for changes in H occurs. Wohlwill (1965) and Harway (1963) altered station points by having their subjects move from one height to another. Distance judgments were unaffected. This suggests, then, that if the subject knows that $\mathrm{H}$ has changed, a compensation can be made. If the $\mathrm{S}$ does not know that changes in $\mathrm{H}$ have occurred, such changes affect distance 
perception. This can be easily demonstrated by photographing the same scene from varying heights. The effect of $\mathrm{H}$ on distance underlies the established photographic principle (Adams, 1970) that elevating the camera increases apparent distance from the viewer. These data and demonstrations show that optical gradients alone provide an effective informational basis for the perception of slant and relative distance, but that absolute distance judgment requires registration of height, as Purdy's equations demonstrate.

Motion-carried information has also been found to affect space perception. Schiff (1965) found that the optical expansion pattern specifying looming elicited avoidance in crabs, frogs, and chicks and that opticaily specified time to collision affected response latency. Similarly, Ball and Tronick (1971) and Bower, Broughton, and Moore (1971) have found that human infants, including neonates, can distinguish between impending collision and a near miss specified by optical information.

A consideration of distance estimation based on motion parallax and motion perspective also reveals which informational parameters can be registered. For relative judgments of distance, comparison of array velocities is the minimal ability required. For example, if one fixates Point $A$ and is to judge the relative distance of Point $B$, B's velocity vector will be opposite in sign to that of the observer's motion if $\mathbf{B}$ is nearer and will have the same sign as the observer's motion if $B$ is farther. Experiments such as that by Bourdon (cited in Johansson, 1973) indicate that this simple ability is present.

As Eq. 3 shows, observer velocity must be known for absolute distance judgments to be made. Since V is not specified optically, the ability to make absolute judgments would demonstrate the role of nonvisual information. Rosinski (unpublished), Eriksson (unpublished), and Gogel (cited in Johansson, 1973) have been unable to demonstrate absolute distance perception over a range of 2-7 m. Recently, however, Johansson (1973) has found highly accurate absolute judgments over distances of $30-240 \mathrm{~cm}$, allowing head movement of $20 \mathrm{~mm} \mathrm{sec}-1$. This result suggests that array motion and information for head movement are combined to arrive at absolute distance. Johansson argues that the kinesthetic and visual inputs are integrated into a perceptual system in Gibson's (1966) meaning.

These studies on texture gradient and motioncarried information demonstrate that the parameters defined by Gibson and his colleagues do affect perception.

\section{LIMITATIONS OF THE THEORY}

In a footnote, Eriksson points out that Gibson has concentrated on the perception of relative distance. The reason for this is clear, since he has emphasized the role of optical information and since his own work has shown that other forms of information may be important in absolute distance perception. Such an emphasis does not imply, however, that the theory applies only to relative judgments or only to optical information. This latter fact has been made explicitly. Gibson (1966, p. 283) points out that the perceptual system must have each of its inputs related to the other inputs of the system. Further (p. 284), he points out that the kinesthetic, vestibular, haptic, and visual inputs must be correlated over time. Similarly, E. J. Gibson (1969) has argued that the learning of intermodal relations (multimodal invariants) underlies much of perceptual learning and development. The existence of such multimodal effects, then, is scarcely inconsistent with the theory, although the lack of a precise specification of intermodal relationships does limit the theory's applicability.

Although the role of optical and nonoptical information have been considered, two problems with Gibson's theory do exist which were not mentioned by Eriksson. The first problem involves the assumptions underlying the analyses of potential and effective information described above. These analyses assume that actual texture element size is constant or, at least, varies randomly; that surfaces are planar and rigid; and that motion is regular. In order for stimulus information to be effective, the perceptual system must operate under the same assumptions. For example, since a pattern of converging lines potentially specifies surface slant only, if the lines are assumed to be really parallel, perception of slant based on perspective convergence can occur only if the perceptual system assumes parallelism. Otherwise, perspective specifies an infinite number of slant angles of an infinite number of different surfaces.

There are several instances in which these perceptual assumptions override available information for actual layout. For example, in the Ames window demonstration, static outline convergence specifies that the window is slanted if the system assumes that its actual shape is rectangular. The dynamic motion transformation information in rotation specifies the actual slant and direction of rotation. Based on the transformation sequence, a rotating trapezoid should be seen. The perspective convergence, combined with the assumption of rectangularity, overrides the dynamic information to yield an oscillating, elastic, rectangle.

The existence of such effects indicates that constraints exist on registration of input. Such contextual or assumptive constraints have not been considered in Gibson's theory. Aside from directed attention, factors influencing the input function of the system have been ignored. This lacuna further limits the theory's applicability.

A second difficulty arises from Gibson's (1959) postulation of a psychophysical correspondence between stimulus information and percept. With few 
exceptions (involving slant perception), a 1-to-1 relationship between information and perception has not been found. That such correspondence does not exist is well known and need not be documented here (see Epstein \& Park, 1964). This lack of correspondence is caused by a basic limitation in Gibson's theory. The hypothesis of psychophysical correspondence has implicit in it three assumptions: first, that proximal information exists; secondly, that the perceptual system's input function can register this information; and thirdly, that the input and output functions are isomorphic, that is, that the system transfer function is flat.

These assumptions appear to be correct under some conditions (in some experiments on monocular slant yielding high regressions). As the slope of the transfer function deviates from zero, however, psychophysical correspondence will decrease. The facts that: psychophysical correspondences have often not been found, that slant judgments are effected by the angular size of texture elements (Flock, 1965), and that accuracy of size judgments decreases with increasing distance (see Sedgwick, 1973) indicate that the system transfer functions do not have zero slope across their entire range.

Since Gibson makes no provision for the system transfer function in his theory, his theory cannot account for perception based solely on input. It should be noted that the suggestions that Eriksson makes also have this limitation. A theory that considers kinesthetic, vestibular, proprioceptive, and cognitive information is still incomplete without a specification of the transfer functions of each of these systems and a specification of the transfer function of the system that relates these modalities.

\section{SUMMARY}

It is concluded that optical stimulation is sufficient for the perception of the size and distance, but that absolute judgments in arbitrary units require other sources of information. Rather than being an ignored or weak portion of information-based theory, this fact was first pointed out by J. J. Gibson and E. J. Gibson and their colleagues and is an important part of Gibson and Gibson's theory of perception and perceptual learning. This theory provides exact descriptions of the informational parameters of stimulation, the aspects of space they specify, the information that must be extracted from stimulation, the scale factor that must be learned, and the limits of perceptual judgment based on optical information. The lack of independence of certain parameters serves to reduce the specificity of visual information for certain tasks. Such reductions of specificity are explicit in Gibson's theory.

The major limitation of this theory lies not in the specification of potential or effective information, but in a description of the perceptual system's response to this information. A general theory of perception cannot be based solely on visual and nonvisual information, but must consider the system input and transfer functions as well.

\section{REFERENCES}

Adams, A. Camera and lens. New York: Morgan \& Morgan, 1970. BALL, W.. \& Tronick, E. Infant responses to impending collision: Optical and real. Science. 1971, 171, 818-820.

Bower, T. G. R., Broughton, J. M., \& Moore, M. K. Infant responses to approaching objects: An indicator of response to distal variables. Perception \& Psychophysics, 1971, 9. 193-196.

Braunstein, M. L. Motion and texture as sources of slant information. Journal of Experimental Psychology, 1968, 78, 247-253.

Epstein, W., \& PARK, J. Examination of Gibson's psychophysical hypothesis. Psychological Bulletin, 1964, 62, 180-196.

ErIKSSON. E. S. Distance perception and the ambiguity of visual stimulation: A theoretical note. Perception \& Psychophysics, 1973, 13, 379-381.

FLock, H. R. A possible optical basis for monocular slant perception. Psychological Review, 1964, 71, 380-391.

Flock, H. R. Optical texture and linear perspective as stimuli for slant perception. Psychological Review, 1965, 72, 505-514.

Flock, H. R., \& Moscattelli, A. Variables of surface texture and accuracy of space perception. Perceptual \& Motor Skills, 1964, 19. 327-334.

GiBSON, E. J. Principles of perceptual learning and development. New York: Appleton-Century, 1969.

GiBson, E. J., \& BERGMAN, R. The effect of training on absolute estimation of distance over the ground. Journal of Experimental Psychology, 1954, 48, 473-482.

GiBson, J. J. The perception of the visual world. Boston: Houghton Mifflin, 1950.

GiBson, J. J. Perception as a function of stimulation. In S. Koch (Ed.), Psychology: A study of a science. Vol. 1. New York: McGraw-Hill, 1959. pp. 456-501.

GiBson, J. J. The senses considered as perceptual systems. Boston: Houghton Mifflin, 1966.

Gibson, J. J., Olum, P., \& Rosenblatt, F. Parallax and perspective during aircraft landings. American Journal of Psychology, 1955, 68, 372-385.

HARWAY, N. Judgment of distance in children and adults. Journal of Experimental Psychology, 1963, 65, 385-390.

Johansson, G. Monocular movement parallax and near-space perception. Perception. 1973, 2, 135-146.

Levine, N.. Rosinski, R. R., \& MCDowell, E. Texture gradients in children's perception of surface slant. Paper presented at Eastern Psychological Association, May 1973.

PURdY, W. C. The hypothesis of psychophysical correspondence in space perception. General Electric Technical Information Series, No. R60ELC56, 1960.

SCHiff, W. The perception of impending collision: A study of visually directed avoidant behavior. Psychological Monographs, 1965, 79, Whole No. 604.

SEDGwick, H. The visible horizon: A potential source of visual information for the perception of size and distance. Unpublished doctoral dissertation, Cornell University, 1973.

WoHlwill, J. F. Texture of the stimulus field and age as variables in the perception of relative distance in photographic slides. Journal of Experimental Child Psychology, 1965, 2, 163-177.

(Received for publication October 17, 1973; revision received April 15, 1974.) 